Self, Motivation, and Virtue or: How We Learned to Stop Worrying and Love Deep Integration

\author{
Moin Syed ${ }^{1}$, Colin G. DeYoung ${ }^{2}$, Valerie Tiberius ${ }^{3}$ \\ University of Minnesota
}

Version Date: 01/08/18

This is a draft of a chapter to appear in a collection edited by Nancy Snow and Darcia Narvaez for the Self, Motivation, and Virtue Project, supported by a grant from the Templeton Religion Trust.

Department of Psychology, University of Minnesota, moin@umn.edu Department of Psychology, University of Minnesota, cdeyoung@umn.edu Department of Philosophy, University of Minnesota, tiberius@umn.edu 


\begin{abstract}
The Self, Motivation, and Virtue Project (Snow \& Narvaez, 2015) was developed "to open avenues of inquiry into virtue using the framework of the 'self,' instead of 'personality,' to require deep and ongoing interdisciplinary collaboration, and to stimulate methodological innovation into the study of virtue." But what does this mean to use a "framework of self" instead of "personality?" What do we mean by "self” and "personality"? In this chapter we provide a conceptual review and integration of self and personality, as well as identity, motivation, virtue, and well-being. In clarifying these different concepts, we demonstrate that the concept of personality--when conceptualized as extending beyond personality traits--provides the broadest, most inclusive framework for understanding self, identity, motivation, virtue, as well as all other traits and characteristic adaptations.
\end{abstract}

Keywords: self, personality, identity, well-being 
The Self, Motivation, and Virtue Project (Snow \& Narvaez, 2015) was developed "to open avenues of inquiry into virtue using the framework of the 'self,' instead of 'personality,' to require deep and ongoing interdisciplinary collaboration, and to stimulate methodological innovation into the study of virtue." But what does this mean to use a "framework of self" instead of "personality?” What do we mean by "self” and "personality”? This is a difficult distinction to make succinctly, as the two terms are not used consistently across psychology and associated disciplines.

In this chapter, we review the major inconsistencies in the use of "self” and "personality," as well as "identity," which is another key concept that is not often clearly distinguished from self and personality. The thrust of our argument is that the concept of personality--when conceptualized as extending beyond personality traits--provides a broad framework for understanding not only self and identity, but also consistent individual differences in motivation and virtue.

Additionally, our own interest in these issues arose out of concerns about how different aspects of personality are relevant for well-being. However, "well-being” is another term that is conceptualized and studied in divergent ways, especially when moving beyond psychological conceptions. We review these different perspectives following our discussion of self, identity, personality and suggest that understanding well-being in the context of individuals' personal projects (i.e., active goals) provides one possible strategy for finding common ground among different conceptions.

We approach these issues from our individual perspectives as a developmental psychologist, a personality psychologist, and a philosopher. Perhaps because the philosopher among us takes David Hume as her inspiration, we found that differences between the two psychologists from different sub-fields were as significant as the differences between the psychologists and the philosopher. Hume was an empiricist philosopher who attempted to locate the self, virtue, and indeed all of ethics in a naturalistic picture of human psychology. The Humean tradition in philosophy is, therefore, more amenable to integration with psychology than other philosophical traditions. Nevertheless, we did have three different disciplinary perspectives that led to different assumptions and understandings of self, identity, personality, and well-being. We have not always agreed on all of the issues presented in this chapter, and in some ways still do not, but through collaboration we worked towards a deep integration that has refined our understanding. We highlight some of these challenges to deep integration throughout the chapter.

\section{The Self and Identity}

Few concepts in the social sciences are as confused and diffuse as the "self." Even looking within the field of psychology there is no agreed upon definition of the self and thus the term is not consistently used. Accordingly, a critical starting point for understanding the relations among self, motivation, and virtue is gaining clarity on what, exactly, is the "self."

William James (1890), Charles H. Cooley (1902), and George Herbert Mead (1934) are often credited with laying the foundations for psychological understandings of self and identity. Importantly, even these earliest articulations of the self eschewed a singular global concept of 
"the self" in favor of more specific aspects of the self. For example, James made a distinction between the "me" self and the "I" self. The "me" is the self as object, what is consciously known about the self (and which he further divided into the material self, social self, and spiritual self). In contrast, the "I" is the self as subject, the knower of who one is.

Although both James and Mead emphasized the role of internal and social/interactional processes, James relatively favored the internal whereas Mead relatively favored the social/interactional. This crude alignment is useful for understanding the subsequent intellectual lineages of these two early influential figures as researchers sought to develop formal theories, experiments, and assessments that followed from their ideas (see Hammack, 2015). Mead's work went on to heavily influence sociological and social psychological concepts of the self, which tend to emphasize the nature of the self within specific social contexts. In contrast, James' work was heavily influential in developmental psychological concepts of the self, which tend to emphasize internal processes of continuity, coherence, and person-environment fit. Below we briefly review each of these broad perspectives.

\section{Social Psychological Approaches to Self and Identity}

Although the self is a concept widely used in social psychology, it is not used consistently or coherently. In their introduction to the Handbook of Self and Identity, Leary and Tangney (2012) organize definitions of the self into five categories: 1) self as the total person, 2) self as personality, 3) self as experiencing subject (I self, or self as subject), 4) self as beliefs about oneself (me self, or self as object), and 5) self as executive agent (self as cybernetic system). They reject the first two definitions, largely because those definitions rely on a global "self" that they feel is not specific enough to be useful. The latter three definitions, they argue, can be synthesized into a higher order process of reflexive thinking, or "the ability to take oneself as the object of one's attention and thought" (p. 6) with the self then being defined as "the set of psychological mechanisms or processes that allows organisms to think consciously about themselves” (p. 6).

One confusion that arises with this synthesized definition of the self stems from what seems to be a misunderstanding of two distinct linguistic functions for the word "self." "Self" can refer to a distinct entity, typically a psychological entity such as the conscious experience and understanding of the individual's own being as a coherent agent (encompassing the I and the me, as we have discussed above, and aligning with definitions 3 and 4 above). However, it can also simply signify reflexivity, as it often does when used as a prefix, as in the terms "selfregulation" and "self-control” (aligning with definition 5). Many social psychologists seem to take the existence of these terms as evidence that the "self" as a psychological entity is somehow necessary for inhibiting impulses--as if it were the "self" that was doing the regulation or exercising control. Instead, however, the prefix "self" here is simply indicating reflexivity. Human being are systems that regulate themselves, also known as "cybernetic" systems (DeYoung, 2015). From our perspective, therefore, the fifth definition offered above should also be excluded from the meaning of "self" because, in fact, the whole person is a cybernetic system.

In the context of this linguistic analysis, the case of "self-esteem" is interesting because "self" in this term can be interpreted either as a psychological entity or as a signifier of 
reflexivity. In the latter case, the individual esteems (evaluates) itself. In the former, it is precisely the conscious understanding of what one is that is being evaluated. In this case, the two meanings of "self" are largely congruent; however, in many cases (as with "self-regulation" and "self-control”) they are not, and we observe that this has given the social psychological definition of self a rather amorphous and confused quality. Many unconscious processes are involved in self-regulation and self-control, and we do not think that these should be considered part of the "self" as that term is typically used in psychology. Thus, from our perspective, the self should be limited to the self as experiencing subject and the self as beliefs about oneself, thereby remaining largely congruent with James’ original definitions of the I self and the me self.

One peculiar phrasing commonly used in social psychology is "self and identity." There is the Handbook of Self and Identity, but there is also the journal Self and Identity, which is the flagship journal of the International Society of Self and Identity. The frequent use of both terms suggests that they are believed to be different in some way, but to the best of our knowledge there is no meaningful distinction between them to be made. In their introductory chapter to the Handbook, Leary and Tangney (2012) devote considerable attention to the definitional and conceptual thicket of the self, but do not even offer a definition of identity, let alone how it may differ from self.

Social psychological approaches to identity have largely focused on group-based processes, heavily influenced by social identity theory. Tajfel (1981) defined social identity as "that part of an individual's self-concept which derives from his knowledge of his membership of a social group (or groups) together with the value and emotional significance attached to that membership” (p. 255; italics in original), and the concept has been primarily used to understand situational in-group and out-group behavior (Ashmore, Deaux, \& McLaughlin-Volpe, 2004; Verkuyten, 2016).

Tajfel's definition referenced above is frequently cited in the literature verbatim, often followed by a paraphrase of the contrasting definition of personal identity as the focus on aspects of the self that individuals believe make them unique. But Tafjel did not provide a definition of personal identity, and was quite clear that there was much more to identity than he was considering with social identity. Nevertheless, social psychology has essentially defined the conceptual universe of identity as group memberships (social, or collective, identities) that connect individuals to others, and as personal identities, which are characteristics that make individuals unique from others (e.g., Ashmore et al., 2004; Brewer, 1991).

\section{Developmental Psychological Approaches to Self and Identity}

From the perspective of developmental psychology there is no meaningful difference between self and identity. The lack of distinction between the terms is largely due to convention rather than researchers in the field actively sorting out the differences in the meaning of the terms. When "self" is used by developmental psychologists it is typically applied to individual reflective processes occurring prior to adolescence (e.g., mirror self-recognition in infancy; Suddendorf \& Butler, 2013). Following Erik Erikson (1950), developmental psychologists tend to understand identity as arising in early adolescence in response to broadening cognitive capacities that facilitate deeper reflective thinking and an expanding social milieu that affords 
greater social comparison (Habermas \& Köber, 2015; Meeus, 2011). Thus, the distinction, if any, corresponds to one of maturity or sophistication rather than conceptually distinct psychological phenomena.

The use of the terms “social identity” and "personal identity” in social psychology are quite different from how they are used in developmental psychology. Indeed, the whole concept of identity is understood and studied from a very different perspective (Syed, Azmitia, \& Cooper, 2011). The developmental perspective on identity is rooted in Erikson's $(1950,1968)$ psychosocial theory of lifespan development, which as noted, was heavily influenced by William James' conceptualization of the self. In particular, Erikson was taken by the concept of the I self, exploring individuals' efforts to develop a stable internal sense of self, or identity. For Erikson identity corresponds to a sense of inner sameness, coherence, and integration of the self across time and place.

Erikson also used the terms personal identity and social identity, as well as ego identity, but he used them in a different way than how social psychology has defined them. Erikson conceptualized personal identity as individuals’ personal beliefs and goals in relation to culturally-relevant roles. Social identity was defined in a similar way, as connections individuals have to various group memberships, but also connects identity processes to larger social structures, taking into consideration how individuals develop identities within a specific cultural context (Way \& Rogers, 2015). So both personal and social identities correspond to the me self. Finally, ego identity represents the synthesizing process used to establish and maintain personal continuity, closely aligning with James' conceptualization of the I self. Whereas the social identity perspective largely sets aside the concept of personal identity in favor of exploring social identity, the developmental perspective views the two as closely linked; that personal and social identities can work together to enhance or constrain individual development.

The social and developmental approaches to self and identity have largely operated as parallel streams of research, although this has been more extreme in the social psychological literature. For example in Leary and Tangney's (2012) review of the history of self and identity in psychology there is no reference at all to Erikson's theory or the wealth of empirical research that has followed it. Even when discussing the relevance of psychoanalytic theory for self and identity Leary and Tangney do not invoke Erikson. The only developmental research that is discussed is Harter's (2015; Harter \& Monsour, 1992) research on changes in evaluative traits across adolescence, an approach that is quite consistent with the social psychological study of the self.

To be sure, developmental research has been relatively insular as well, and there are subdivisions within the developmental approach to identity that have mostly failed to adequately interface with one another (i.e., the identity status and narrative approaches, see Syed, 2012; McLean \& Syed, 2015). However, some developmental research, namely in the area of ethnic/racial identity, has explicitly sought to merge the social psychological and developmental perspectives (Phinney, 1990; Umana-Taylor et al., 2014; Verkuyten, 2016; Yip \& Douglass, 2013). 
Despite these many different terms, and identical terms defined in different ways, all uses of self and identity reviewed above conform to Leary and Tangney’s (2012) definition of self as "the set of psychological mechanisms or processes that allows organisms to think consciously about themselves." Accordingly, our position follows the developmental psychologists' view that there is no meaningful distinction between self and identity, which may also be consistent with the social psychological view given the lack of discussion of the distinction. The questions we are now confronted with are: how are self and identity related to personality? What would it mean to use a "framework of self” instead of "personality?"

\section{Self and Identity as Part of Personality}

The major barrier to understanding how self and identity are part of personality is a limited understanding of what personality is. Personality has, unfortunately, come to be equated with personality traits. Personality traits are relatively stable descriptions of individuals' probabilistic tendencies in behavior, motivation, emotion, and cognition. Typically traits are constructs that could be applied to people in any culture at any point in history, rather than being culturally or personally specific (DeYoung, 2015). Traits are a central component of personality, but personality psychologists have never viewed traits as constituting the totality of personality. Rather, traits are viewed as one level of the broader personality structure.

In this section we outline this personality structure and locate self and identity, as used by social and developmental psychologists, within it. Using this framework helps resolve some of the confusion between social and developmental definitions of self and identity, and also helps facilitate an understanding of the cultural nature of self, identity, and personality.

\section{Three Levels of Personality}

Building upon McAdams’ (1995) earlier work, McAdams and Pals (2006) outlined an integrative framework for understanding the complexities of the whole person. This framework highlighted five principles with which personality can be understood, with the middle three principles constituting three levels of personality:

Personality is conceived as (a) an individual's unique variation on the general evolutionary design for human nature, expressed as a developing pattern of (b) dispositional traits, (c) characteristic adaptations, and (d) self-defining life narratives, complexly and differentially situated (e) in culture and social context (p, 204).

Biology and culture are less distinct parts of personality than facts about the nature of human variation; that is, there is always a biological and a cultural foundation underlying any personality process. It is due to evolution that all human beings share a general biological plan for the body and brain, and personality stems from variations in this plan. Similarly, all human beings live within a cultural context that shapes their individual personalities. Thus, we focus on the three interior levels of traits, characteristic adaptations, and life stories. 


\section{Level 1: Traits}

Personality traits refer to relatively stable individual differences in behavior, motivation, emotion and cognition that constitute the most basic, fundamental aspect of personality (Caspi, Roberts, \& Shiner, 2005; Costa \& McCrae, 1992; DeYoung, 2015). Although commonly equated with the "big five" of neuroticism, extraversion, conscientiousness, agreeableness, and openness, personality traits have been more broadly organized into a hierarchical model based on their patterns of covariation. Each of the big five traits subsumes two aspects, and each aspects consists of some unknown number of facets (DeYoung, Quilty, \& Peterson, 2007). Moving up from the big five are the two meta-traits of plasticity, consisting of the shared variance between openness and extraversion, and stability, consisting of the shared variance of conscientiousness, agreeableness, and low neuroticism (i.e., emotional stability). Personality traits that cannot easily be fit into a hierarchy based on the Big Five are nonetheless personality traits, as long as they reflect differences in behavior and experience that can be meaningfully used to describe people across cultures. The Big Five are simply the major dimensions of covariation among the multitude of more specific personality traits. In this context, many of the characteristics that are seen as “virtues" -- compassion, honesty, courage, etc. -- are personality traits.

\section{Level 2: Characteristic Adaptations}

McAdams and Pals (2006) defined characteristic adaptations as "a wide range of motivational, social- cognitive, and developmental adaptations, contextualized in time, place, and/or social role” (p. 208). Fitting with this broad definition, they provided a long list of psychological constructs that are captured by this level, including goals, plans, self-images, and developmental tasks, all while noting that there is no agreed upon taxonomy of characteristic adaptations akin to the hierarchical model of traits. DeYoung (2015) provided a simplified definition of characteristic adaptations, asserting that this aspect of personality can be decomposed into just three broad categories, goals that guide behavior, interpretations of self and world, and cognitive and behavioral strategies used to pursue goals, attempting to transform the state of existence as currently perceived into the one desired.

As communicated by the label characteristic adaptations, this level of personality is more squarely concerned with the persistent ways in which individuals have responded (i.e., adapted) to their specific life circumstances. Although characteristic adaptations are clearly more contextualized than traits, it would not be accurate to distinguish characteristic adaptations and traits as the contextualized vs. decontextualized aspects of personality, as a wealth of research has demonstrated the contextual nature of traits (Roberts \& Caspi, 2003; Roberts et al., 2017; Thorne, 2000). Rather, traits are viewed as culturally universal dimensions of human variation whereas characteristic adaptations are responses to specific cultural, or even uniquely personal, demands. Moreover, the distinction between traits and characteristic adaptations is sometimes described as the "having” vs. the "doing” of personality (e.g., McAdams \& Pals, 2006). This is also not accurate, as traits are also put into action for specific purposes. 


\section{Level 3: Integrative Life Narratives}

Integrated life narratives consist of the stories that people tell about their personal past to make meaning and develop a sense of identity (McAdams \& Pals, 2006). This level of personality evolved from a somewhat different tradition than traits and characteristic adaptations. Narrative psychology focuses on how individuals reason about their past experiences and how stories serve functional roles for self-understanding (McAdams, 2013). Narrating one's life allows for a sense of coherence across time and place to provide a sense of purpose and meaning. In contrast to traits and characteristic adaptations, the integrated life story emphasizes an individual life lived, extended through time. Personality at this level considers the complex and contextual ways in which traits and characteristic adaptations manifest in specific, yet potentially predictable, ways across lifetimes (Josselson \& Flum, 2015). Moreover, integrative life narratives are necessarily situated within individuals' social, cultural, and historical context. Thus, this level of personality aims to understand what is truly unique about a person.

\section{Self vs. Personality}

Having laid out the three levels of personality, we now return to the question, What would it mean to use a "framework of self” instead of "personality?" We believe that the personality structure outlined above actually simplifies all of this a great deal, providing two workable solutions. One solution is to define the self in the global sense and equate it with personality, as Leary and Tangney (2012) indicated is already done in some circles, and then recognize the distinct levels of the self within the complex structure of personality. Within this approach self and personality constitute an instance of the jangle fallacy (two different terms that nevertheless refer to the same thing), and thus use of both terms should be avoided (in this case, cease using the vague term self in favor of personality). This solution fits well with the philosopher David Hume's conception of the self as a "bundle of perceptions". Hume did not have the benefit of research in contemporary personality psychology, but were he around today he likely would acknowledge the different components of personality discussed in the previous section and included them in his "bundle theory". This solution suggests there is no difference between a "framework of self" and personality.

An alternative solution would rely on the more narrow definition of self as referring to psychological mechanisms associated with conscious representation of who one is, which is consistent with developmental and most social psychological definitions of self. The self is one's conscious understanding of what one is as a person, including one's conscious beliefs about what one values, what one is capable of, what one's skills are, and how one orients towards the future. This definition makes the self a part of personality; reflective processes are a subset of the broader class of interpretations, which are themselves characteristic adaptations. Since even under the narrower conception of the self, most aspects of the self reflect levels 2 and 3 of personality (characteristic adaptations and integrative life narratives), it is not necessary to distinguish between a "framework of self" and a "framework of personality." The self (broadly or narrowly defined) is subsumed by the concept of personality. 


\section{Interlude: Defining Personality Levels as a Challenge to Deep integration}

Agreeing on the general structure of personality does not resolve all potential conflicts about how to conceptualize aspects of the personality system. Sorting complex constructs, such as self, identity, motivation, and virtue, into the different levels is certain to lead to disagreements among researchers coming from different traditions.

Identity is a case where we, the authors, do not completely agree. In the McAdams and Pals (2006) framework identity is viewed as a level 3 construct whereas self processes (in the reflexive sense of the term) are viewed as level 2. McAdams and Pals did not make any effort to distinguish between self and identity conceptually, and as we discussed above, there is no meaningful distinction to be made. That said, from Moin's perspective identity can be defined at levels 2 and 3 not because of an artificial distinction between self and identity, but because each level provides a substantially different degree of information and insight into identity as part of personality (Syed, 2017). Identity clearly operates at level 2 with regard to how individuals engage with the specific domains of their identity (e.g., occupation, religion, family) over a relatively circumscribed period of time. Level 3, in contrast, focuses on the synthesis of the self across an extended period of time to create and maintain an overall sense of identity. From Colin's perspective, interpretations of the self arise in response to specific cultural circumstances and are therefore characteristic adaptations. In this sense, integrative life narratives are certainly a key part of the self, but so are less elaborated forms of identity, including self-esteem and one's sense of what one's own traits are. Thus, Colin rejects the ontological distinction between levels 2 and 3 and describes integrative life narratives as simply a special type of characteristic adaptation in the interpretation category (DeYoung, 2015).

Indeed, the source of this disagreement is rooted in a larger disagreement between Colin and Moin about whether levels 2 and 3 should be conceptualized as distinct levels of personality at all, even though they agree that the integrative life narrative is distinguishable from from other types of characteristic adaptations.For Colin, the integrative life narrative is an interpretation of the self, and therefore a characteristic adaptation. For Moin, the integrated life narrative represents what is truly unique about a person and his or her life, and this degree of difference with respect to what we know about a person is sufficiently large so as to justify its status as a distinct level of personality. Colin asserts that there are truly unique aspects of a person's personality at both levels 2 and 3 in the McAdams and Pals framework and hence does not see the uniqueness of the life narrative as adequate justification for making it a separate ontological category.

Following prolonged discussion, we determined that the source of our disagreement is distinguishing between epistemological concerns (Moin's: the importance of integrative narratives as a distinctive way for knowing the personality) and ontological concerns (Colin's: what is the integrative narrative as a psychological entity that the individual produces -- it is an interpretation of self/world and hence a characteristic adaptation). This example is instructive because it highlights the differences that can exist within the discipline of psychology, and how deep integration is needed not only between disciplines, but within disciplines as well. 
And what about motivation and virtue? How do we distribute these concepts in the broader personality system? As it turns out this was a rather straightforward issue for us that did not require any deep integration. We are in agreement that all reasonably persistent psychological qualities are either traits or characteristic adaptations, and many qualities can be considered traits or characteristic adaptations, depending on their specific conceptualization and measurement (DeYoung, 2015).

With respect to virtue, we presume that both traits and characteristic adaptations can be virtues, and that every virtue is either a trait or a characteristic adaptations (although clearly not every trait or characteristic adaptations is a virtue). More specifically, virtue can be considered a quality that traits and characteristic adaptations may have, and a virtuous trait or characteristic adaptations can, therefore, be described as a "virtue." Many virtues — honesty, perseverance, kindness, courage, etc.- - are obviously traits. Variation in them could be observed in any culture, and almost any virtuous trait can be classified within the hierarchical taxonomy provided by the Big Five (or as a blend of two or more traits from the hierarchy). That said, not all virtues are traits; one may identify culturally specific virtues — for example, having the skill or motivation to carry out some particular social tradition, or to express a virtuous trait through some specific means - that are clearly characteristic adaptations. Importantly, this conceptualization of how virtues can be traits or characteristic adaptations is not unique to virtues and can easily be extended to motivation.

What defines a particular psychological quality as a trait or characteristic adaptation has little to do with its kind (e.g., motivational, emotional, or cognitive process) and more about its specific conceptualization and measurement. Again, contemporary followers of Hume will find this picture very sympathetic. Hume defined virtues as qualities that are useful or agreeable to the self or others. Reading the findings of personality psychology back into Hume, he could easily agree that virtues are traits or characteristic adaptations that are singled out as virtues because of their positive effects on the possessor of the virtue or on other people.

\section{Deep Integration Continued: What Does all of this Mean for Well-being?}

As noted at the outset of this chapter, we approached our collaboration with a mutual interest in how personality is associated with well-being. Thus far we have focused on different conceptualizations of self, identity, and personality, but we also tend to work from slightly different conceptualization of well-being. As it turns out, however, our different conceptualizations were more similar than different.

One distinctive feature of well-being is that it is good for the creature who has it. In other words, it is a kind of value that is subject relative (Sumner 1996). Psychological theories of wellbeing account for subject-relativity directly by holding that well-being just is a subjective state: it consists in feeling a certain way, or having certain attitudes toward one's life. Indeed, some psychologists (e.g., Diener, Lucas, \& Oishi, 2002) restrict their attention to subjective well-being and do not claim to be studying well-being simpliciter.

Philosophers who aim to describe well-being (not merely "subjective well-being”) have taken different approaches to accounting for the subject relativity of well-being. Some have 
defined well-being in terms of psychological states like pleasure (Feldman 2004, Crisp 2006) or life satisfaction (Sumner 1996). Others have defended objective theories of well-being, according to which there are standards for what counts as good for a person that transcend the person him or herself (Bloomfield 2014, Fletcher 2013). Still others have defended theories of well-being according to which well-being consists in obtaining the objects of our desires, achieving our goals, or fulfilling our values (Heathwood 2016, Keller 2004, Tiberius 2008).

The notion of subjective well-being favored by many psychologists has much in common with the hedonist's definition of well-being but does not offer much of obvious interest to other philosophical schools (Tiberius 2006). This is a serious obstacle to interdisciplinary research and one that we hoped to overcome. Happily, the philosopher on our team defends a value fulfilment theory of well-being, and the cybernetic theory of agency that informs DeYoung's personality research suggests a theory of well being in the family of theories that include value fulfillment and goal achievement theories. This view is also consistent with Syed's view of well-being rooted in developmental psychology, which often conceptualizes well-being as the successful resolution of normative developmental tasks (e.g., developing a sense of identity, forming and maintaining intimate relationships).

This, together with considerations about available measurement tools, led us to think of well-being in terms of success in personal projects. Personal projects are "extended sets of personally salient action in context" (Little, 2006). They are extended in that they take some time (days, weeks, even years) to carry out. They involve some goal that is personally salient-that is, reasonably important to the individual-along with its various subgoals, the strategies that are used to achieve those goals, and the interpretations of the relevant aspects of self and world that allow people both to define the context in which the goals are pursued and to judge when the goals have been met. Researchers who defend different theories of well-being will differ over whether success in personal projects is intrinsically good or good because it leads to something else (such as pleasure) and whether the relevant projects must be subjectively or objectively desirable, but few would deny that the fulfillment of at least some valued personal projects is a significant aspect of well-being.

This approach had the significant advantage that those who do not accept a goal fulfillment theory of the nature of well-being may nevertheless be interested in the results of the research. This is because personal projects include many things that philosophers who defend objective theories will consider to be objectively valuable (such as relationships, acquiring knowledge, and developing skills).

\section{Conclusion}

In this chapter we drew upon our interdisciplinary collaboration to provide a conceptual clarification of self, identity, and personality, and how they fit together. Our discussion was framed around the central focus of the Self, Motivation, and Virtue Project of using a "framework of self" instead of "personality" to understand motivation, virtue, and well-being. In clarifying these different concepts, we demonstrated that the concept of personality--when conceptualized as extending beyond personality traits--provides the broadest, most inclusive framework for understanding self, identity, motivation, virtue, as well as all other traits and 
characteristic adaptations. Additionally, we have highlighted how bringing together scholars with diverse perspectives can lead to increased clarity of terms and concepts, which in turn can lead to greater scientific progress. 


\section{References}

Ashmore, R. D., Deaux, K., \& McLaughlin-Volpe, T. (2004). An organizing framework for collective identity: articulation and significance of multidimensionality. Psychological Bulletin, 130(1), 80-114.

Bloomfield, P. (2014), The Virtues of Happiness: A Theory of the Good Life. New York: Oxford University Press.

Brewer, M. B. (1991). The social self: On being the same and different at the same time. Personality and Social Psychology Bulletin, 17(5), 475-482.

Caspi, A., Roberts, B. W., \& Shiner, R. L. (2005). Personality development: Stability and change. Annual Review of Psychology, 56, 453-484.

Cooley, C. H. (1902). Human nature and the social order. New York: Scribners.

Costa, P. T., \& McCrae, R. R. (1992). Four ways five factors are basic. Personality and individual differences, 13(6), 653-665.

Crisp, R. (2006), “Hedonism Reconsidered”, Philosophy and Phenomenological Research 73 (3): 619-645.

DeYoung, C. G. (2015). Cybernetic big five theory. Journal of Research in Personality, 56, 3358.

DeYoung, C. G., Quilty, L. C., \& Peterson, J. B. (2007). Between facets and domains: 10 aspects of the Big Five. Journal of Personality and Social Psychology, 93, 880-896.

Diener, E., Lucas, R. E., \& Oishi, S. (2002). Subjective well-being: The science of happiness and life satisfaction. In C. R. Snyder \& S. J. Lopez (Eds.), Handbook of positive psychology. New York: Oxford University Press.

Erikson, E. (1950). Childhood and society. New York: W W Norton \& Co.

Erikson, E. (1968). Identity: Youth and crisis. New York: W W Norton \& Co.

Feldman, F. (2004), Pleasure and the Good Life: Concerning the Nature, Varieties and Plausibility of Hedonism. New York: Oxford University Press.

Fletcher, G. (2013), “A Fresh Start for the Objective-List Theory of Well-Being”, Utilitas, 25 (2): 206-220.

Habermas, T., \& Köber, C. (2015). Autobiographical reasoning is constitutive for narrative identity: The role of the life story for personal continuity. In K. C. McLean \& M. Syed 
(Eds.), The Oxford handbook of identity development. New York: Oxford University Press.

Hammack, P. L. (2015). Theoretical foundation of identity. In K. C. McLean \& M. Syed (Eds.), The Oxford handbook of identity development. New York: Oxford University Press.

Harter, S. (2015). The construction of the self: Developmental and sociocultural foundations. Guilford Publications.

Harter, S., \& Monsour, A. (1992). Development analysis of conflict caused by opposing attributes in the adolescent self-portrait. Developmental Psychology, 28(2), 251-260.

Heathwood, C. (2016), "Desire-Fulfillment Theory", in G. Fletcher (ed.) The Routledge Handbook of Philosophy of Well-Being. Oxford and New York: Routledge: 135-147.

James, W. (1890). The principles of psychology. New York: Holt.

Josselson, R., \& Flum, H. (2015). Identity status: On refinding the people. In K.C. McLean \& M. Syed (Eds.), The Oxford handbook of identity development. New York: Oxford University Press.

Keller, S. (2004), "Welfare and the Achievement of Goals", Philosophical Studies, 121 (1): 2741.

Leary, M. R., \& Tangney, J. P. (2011). The self as an organizing construct in the behavioral and social sciences. In M. R. Leary \& J. P. Tangney (Eds.), Handbook of self and identity (pp. 1-20). New York: Guilford Press.

Little, B. R. (2006). Personality science and self-regulation: Personal projects as integrative units. Applied Psychology: An International Review, 55, 419-427.

McAdams, D. P. (1995). What do we know when we know a person? Journal of Personality, 63(3), 365-396.

McAdams, D. P. (2013). The psychological self as actor, agent, and author. Perspectives on Psychological Science, 8(3), 272-295.

McAdams, D. P., \& Pals, J. L. (2006). A new Big Five: Fundamental principles for an integrative science of personality. American Psychologist, 61, 204-217.

McLean, K. C., \& Syed, M. (2015). The field of identity development needs an identity: Introduction to the handbook of identity development. In K. C. McLean \& M. Syed (Eds.), The Oxford handbook of identity development. New York: Oxford University Press.

Mead, G. H. (1934). Mind, self and society. Chicago: University of Chicago Press. 
Meeus, W. (2011). The study of adolescent identity formation 2000-2010: A review of longitudinal research. Journal of Research on Adolescence, 21, 75-94.

Phinney, J. S. (1990). Ethnic identity in adolescents and adults: review of research. Psychological Bulletin, 108(3), 499-514.

Roberts, B. W., \& Caspi, A. (2003). The cumulative continuity model of personality development: Striking a balance between continuity and change in personality traits across the life course. In U.M.Staudinger \& U. Lindenberger (Eds.), Understanding human development (pp. 183-214). Boston, MA: Springer.

Roberts, B. W., Luo, J., Briley, D. A., Chow, P. I., Su, R., \& Hill, P. L. (2017). A systematic review of personality trait change through intervention. Psychological Bulletin, 143(2), 117-141

Snow, N., \& Narvaez, D. (2015). SMV project detailed overview. Retrieved from http://smvproject.com/about/overview/.

Suddendorf, T., \& Butler, D. L. (2013). The nature of visual self-recognition. Trends in Cognitive Sciences, 17(3), 121-127.

Sumner, L. (1996), Welfare, Happiness, and Ethics. Oxford: Clarendon Press.

Syed, M. (2012). The past, present, and future of Eriksonian identity research: Introduction to the Special Issue. Identity: An International Journal of Theory and Research, 12(1), 1-7.

Syed, M. (2017). Advancing the cultural study of personality and identity: Models, methods, and outcomes. Current Issues in Personality Psychology, 5(1), 65-72.

Syed, M., Azmitia, M., \& Cooper, C. R. (2011). Identity and academic success among underrepresented ethnic minorities: An interdisciplinary review and integration. Journal of Social Issues, 67(3), 442-468.

Tajfel, H. (1981). Human groups and social categories: Studies in social psychology. Cambridge: Cambridge University Press.

Thorne, A. (2000). Personal memory telling and personality development. Personality and Social Psychology Review, 4(1), 45-56

Tiberius, V. (2006). Well-being: Psychological research for philosophers. Philosophy Compass, 1, 493-505.

Tiberius, V. (2008), The Reflective Life: Living Wisely with Our Limits. New York: Oxford University Press. 
Umaña-Taylor, A. J., Quintana, S. M., Lee, R. M., Cross, W. E., Rivas-Drake, D., Schwartz, S. J., Syed, M., Yip, T., Seaton, E., \& Ethnic/Racial Identity Study Group. (2014). Ethnic and racial identity revisited: An integrated conceptualization. Child Development, 85(1), 21-39.

Verkuyten, M. (2016). Further Conceptualizing Ethnic and Racial Identity Research: The Social Identity Approach and Its Dynamic Model. Child Development, 87(6), 1796-1812.

Way, N., \& Rogers, O. (2015). “[T]hey say Black men won’t make it, but I know I’m gonna make it”: Ethnic and racial identity development in the context of cultural stereotypes. In K. C. McLean \& M. Syed (Eds.), The Oxford handbook of identity development. New York: Oxford University Press.

Yip, T., \& Douglass, S. (2013). The application of experience sampling approaches to the study of ethnic identity: New developmental insights and directions. Child Development Perspectives, 7(4), 211-214. 